\title{
Reliability of Aerosol Jet Printed Fluorescence Quenching Sensor Arrays for the Identification and Quantification of Explosive Vapors
}

Nico Bolse, ${ }^{*}+\oplus$ Ralph Eckstein, ${ }^{\dagger, \dagger}$ Anne Habermehl, ${ }^{\dagger}$ Gerardo Hernandez-Sosa, ${ }^{\dagger, \oplus}$ Carsten Eschenbaum, ${ }^{\dagger,+, \S}$ and Uli Lemmer ${ }^{\dagger,+, \S \odot}$

${ }^{\dagger}$ Light Technology Institute, Karlsruhe Institute of Technology (KIT), Engesserstrasse 13, 76131 Karlsruhe, Germany

${ }^{\ddagger}$ InnovationLab GmbH, Speyerer Str. 4, 69115 Heidelberg, Germany

${ }^{\S}$ Institute of Microstructure Technology, Karlsruhe Institute of Technology (KIT), Hermann-von-Helmholtz-Platz 1, 76344

Eggenstein-Leopoldshafen, Germany

\section{Supporting Information}

ABSTRACT: One of the primary challenges in explosive detection using fluorescence quenching is the identification and quantification of detected targets. In this work, we explore the reliability of aerosol jet printed sensor arrays for the discrimination of nitroaromatic traces using linear discriminant analysis (LDA). We varied the amount of the deposited material by controlling the printer's shutter to investigate the impact on the detection reliability. For a twofold variation of the amount of the deposited material, we report excellent classification rates between 81 and $96 \%$ for the discrimination of nitrobenzene, 1,3-dinitrobenzene, and 2,4-dinitrotoluene at 1,3 , and 10 parts per billion in air, respectively. Our results

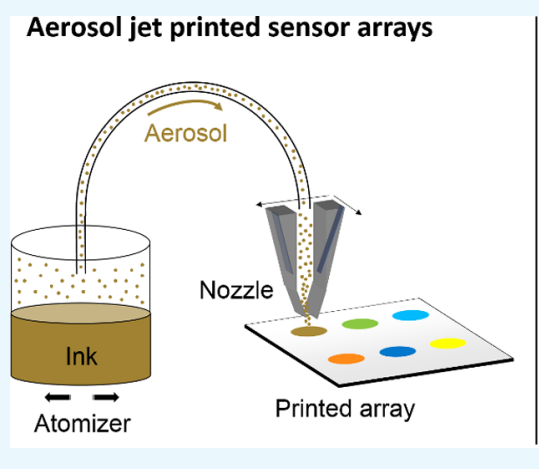
Explosives detection close to the detection limits indicate a remarkable identification and quantification of explosive trace vapors because of high control of the printing process. This work demonstrates the high potential of digitally printed fluorescence quenching sensor arrays and the excellent capabilities of LDA as a simple supervised statistical learning technique.

\section{INTRODUCTION}

The detection of nitroaromatic explosives has become increasingly important since terrorist activities raised security concerns worldwide. ${ }^{1-5}$ To this end, fluorescence quenching transducers offer outstanding sensitivities. ${ }^{6-10}$ Among other materials such as dendrimers and metal-organic frameworks, fluorescent polymers enable the detection of nitroaromatic vapors at concentrations down to parts per trillion in air. ${ }^{11-19}$ Therefore, the utilization of fluorescence quenching in sensors offers the potential to outperform the dog's nose by almost 3 orders of magnitude. ${ }^{20-23}$

Moreover, this approach enables the realization of low-cost systems because of the detection principle requiring only three technical components in the simplest case: an excitation source such as an ultraviolet light-emitting diode (UV-LED), a transducing fluorescent emitter, and a photodetector. A single-peak fluorophore, however, cannot discriminate target analytes because of the lack of selectivity in the transduction mechanisms. In fact, the ability to discriminate targets requires sensor arrays with several differently sensitive emitters or single fluorophores with multiple emission peaks to enable the identification and quantification of multiple target analytes using pattern recognition techniques. ${ }^{24-33}$ However, this approach remains challenging because pattern recognition techniques require intensive training to establish reliable discrimination models for a certain class of target analytes. Sensor drift and reproducibility issues make the discrimination of detected target analytes even more difficult. Therefore, sensor arrays for field applications require stability and a reproducible, robust, and thus reliable process for sensor array fabrication.

Previously, we demonstrated the feasibility of aerosol jet (AJ) printed fluorescence quenching sensor arrays for the discrimination of explosive vapors and their reproducible fabrication within a batch. ${ }^{34,35}$ In this work, we introduce artificially generated fabrication tolerances to explore the reliability of the printing process even further. To this end, we vary the printer nozzle's shutter time in the AJ printing process to investigate the influence of the amount of the deposited material on the detection reliability and on the sensor array reproducibility. The amount of the deposited material has a direct impact on the sensor array response because of the thickness dependence of fluorescence quenching. Hence, we have fabricated three sets of sensor arrays with two- and fourfold variations of the amount of

Received: August 28, 2017

Accepted: September 12, 2017

Published: October 9, 2017 
the deposited material to evaluate their reliability in terms of discriminatory reproducibility for three nitroaromatic compounds at parts-per-billion ( $\mathrm{ppb}$ ) concentrations in air.

\section{EXPERIMENTAL SECTION}

Each sample set consists of 12 sensor arrays ( $4 \mathrm{~mm}$ pitch) with 6 different polymer pixels $(0.5 \mathrm{~mm}$ pitch). Four of the polymers were received from Merck KGaA including PDY-132 (SY), SPG-01T (PG), SPB-02T (PB), and SPW-111 (PW). The other two polymers ADS229BE (PFO) and ADS133YE (F8BT) were purchased from American Dye Source. Each polymer was dissolved in toluene and tetralin (1:1 by volume) to provide the ink for the AJ printer that was equipped with a $200 \mu \mathrm{m}$ nozzle. Each sample set was printed onto conventional glass substrates that were pretreated in an oxygen plasma for surface activation. The substrates were kept at $50{ }^{\circ} \mathrm{C}$ during printing to control the drying process. The printer nozzle was equipped with a shutter to stop material deposition without stopping the aerosol generation and gas flows. To realize the three different sets of sensor arrays, the printer nozzle's shutter was kept open for $0.5 \mathrm{~s}$ (P1), $1 \mathrm{~s}$ (P2), and $2 \mathrm{~s}$ (P3) for the deposition of each polymer pixel. In comparison to P1, the amount of the deposited material for each polymer pixel doubles and quadruples for P2 and for P3, respectively.

A permeation-based vapor generator (Dynacalibrator 235, VICI) was used for the delivery of constant vapor concentrations in a carrier stream of dry air. Here, the vapors emitted from the permeation tubes containing nitrobenzene (NB), 1,3-dinitrobenzene (DNB), and 2,4-dinitrotoluene (DNT) were used as nitroaromatic target analytes. These compounds are used in the manufacture of common explosives. Vapor concentrations of 1,3 , and $10 \mathrm{ppb}$ in air were delivered by dilution of the analyte carrier flow. The target analytes were used as received from MACHEREY-NAGEL GmbH \& Co. KG. Great care has to be taken when working with these hazardous compounds because of their carcinogenic, mutagenic, and reproduction toxic characters. The fluorescence was excited over the full area of the $26 \times 26 \mathrm{~mm}^{2}$ samples with roughly $6 \mu \mathrm{W}$ using a collimated $365 \mathrm{~nm}$ UV-LED (M365L2, Thorlabs). A charge-coupled device camera (DFK21BU618, The Imaging Source) was used to measure the average fluorescence intensity as detected by $5 \times 5$ camera pixels for each polymer structure. The fluorescence quenching efficiencies (QEs) according to $\mathrm{QE}=\left(I_{0}-I\right) / I_{0}$, with $I_{0}$ as the intensity in the absence of a target analyte and $I$ as the intensity in the presence of it, were averaged over the 12 sensor arrays in each set using the background-corrected fluorescence intensities. A drawing of the experimental setup is depicted in Figure 1.

Linear discriminant analysis (LDA) was used to assess the discrimination of the target analytes at different concentrations for each sample set using Mahalanobis distance-based classifiers. A target class was defined by the QEs observed for each specific concentration and analyte. For these classifications, the accuracy rate describes the relative amount of correctly classified observations when the LDA was trained with one of the three sample sets, whereas the classes for the data from the other two sets were predicted. For this purpose, the LDA input data were standardized. This means that the QEs for each polymer were scaled to have zero mean and standard deviation equal to one.

The molecular structures of the target analytes, the measurement sequence for the fluorescence quenching experiments, the normalized fluorescence signals for each set of

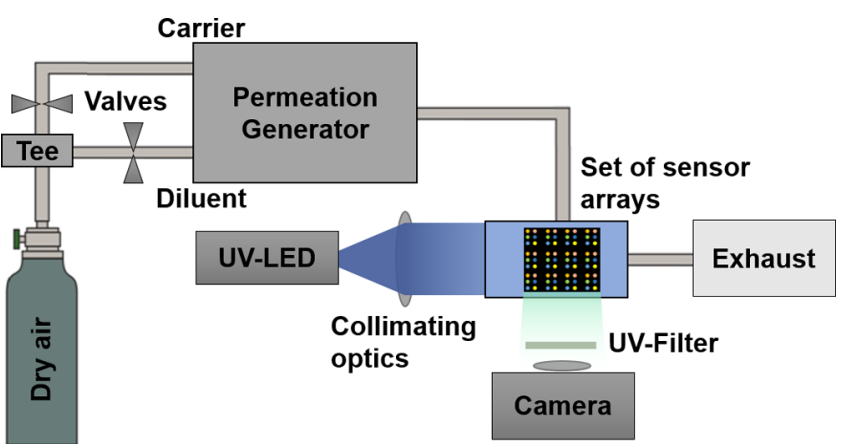

Figure 1. Drawing of the experimental setup showing the permeationbased vapor delivery and the optical setup for fluorescence quenching detection.

sensor arrays, and detailed confusion matrices for the classification tests are provided in the Supporting Information. The photophysical properties of the polymers and details on the sample preparation and on the LDA have been reported elsewhere. $^{34,35}$

\section{RESULTS AND DISCUSSION}

Figure $2 a-c$ shows an image of the three sample sets P1, P2, and P3 under UV illumination. The scanning electron

\section{(a) P1 $4 \mathrm{~mm}$}

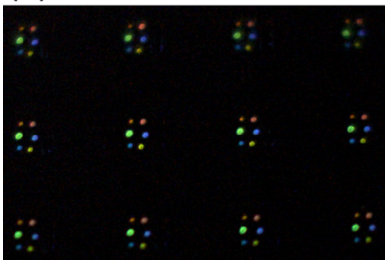

(b) P2

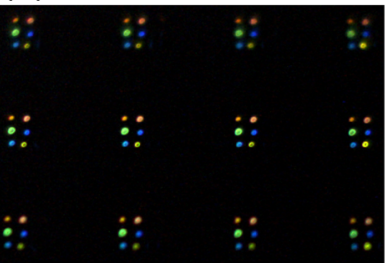

(c) P3

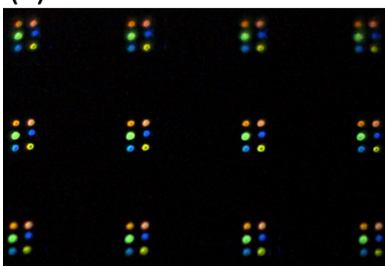

(d)
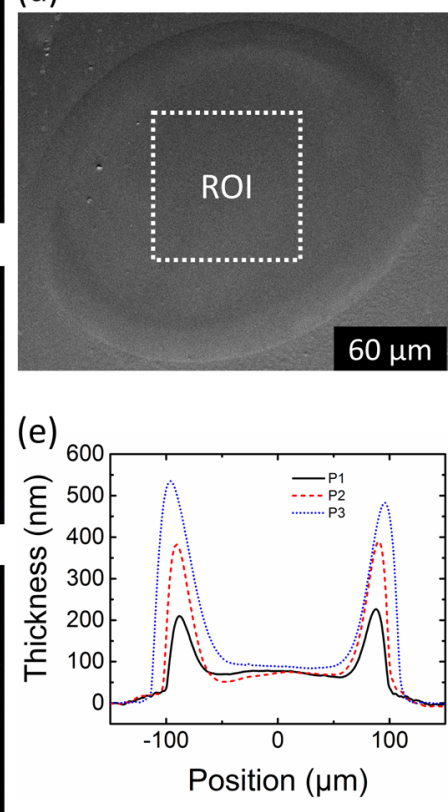

Figure 2. UV-excited sample sets: (a) P1, (b) P2, and (c) P3 as seen by the camera. The fluorescence in each array from top to bottom and from left to right corresponds to SY, PG, PB, PW, PFO, and F8BT. (d) SEM image of a printed structure including ROI indication. (e) Exemplary cross sections.

microscopy (SEM) image in Figure $2 \mathrm{~d}$ and the cross sections of the printed polymer pixels in Figure 2e reveal a circular structure with a homogeneous plateau in the center and an outer ring with higher thickness. This type of structure is a result of the drying ink. The centered plateau serves as the region of interest (ROI) to measure the fluorescence. An 
increase in the nozzle's shutter time from P1 over P2 to P3 leads to a brighter appearance of each pixel.

Table 1 shows the increasing average brightness of each polymer fluorescence for increasing shutter time. By contrast,

Table 1. Mean Absolute Fluorescence Intensities for Each Polymer As Measured in Air

\begin{tabular}{clll} 
& \multicolumn{3}{c}{ absolute fluorescence intensity (arb unit) } \\
\cline { 2 - 4 } polymer & P1 & P2 & P3 \\
SY & 73 & 114 & 147 \\
PG & 160 & 171 & 184 \\
PB & 66 & 92 & 94 \\
PW & 121 & 152 & 161 \\
PFO & 82 & 86 & 87 \\
F8BT & 107 & 152 & 186 \\
\hline
\end{tabular}

we expect a constant brightness because of the location of the ROIs within the centered plateau whose thickness is independent of the shutter time. The increasing fluorescence, however, is a result of the crosstalk from the outer ringlike structures such that they contribute fluorescence to the ROIs within the centered plateaus. The outer rings become slightly broader and increase in height from roughly $200 \mathrm{~nm}$ (P1) over $400 \mathrm{~nm}$ (P2) up to $500 \mathrm{~nm}$ (P3), as can be seen in Figure 2e.

We performed long-term illumination experiments to study the optical degradation in terms of photobleaching. For this purpose, each sample set resides in a stream of dry air at $1 \mathrm{~L} /$ min for a duration of $4 \mathrm{~h}$ under a $6 \mu \mathrm{W}$ illumination power over the full sample area. Figure 3 shows the measured degradation

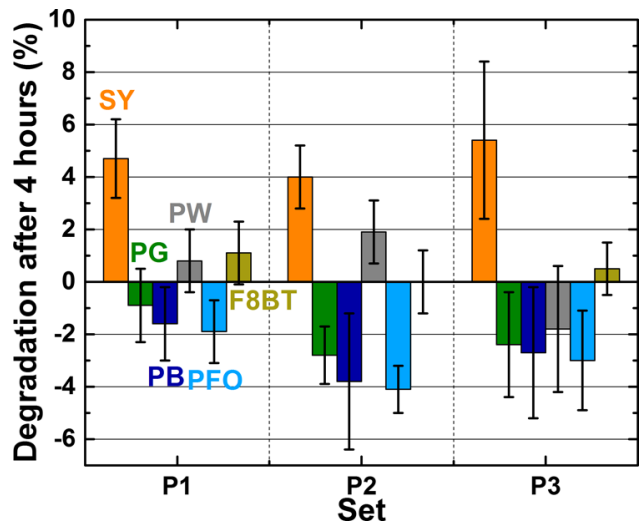

Figure 3. Optical degradation of the polymer fluorescence after being excited with $6 \mu \mathrm{W}$ across the full sample area for a duration of $4 \mathrm{~h}$ in a stream of dry air at $1 \mathrm{~L} / \mathrm{min}$. For each polymer pixel, the error bars represent the standard deviation across the 12 structures in each sample set.

after $4 \mathrm{~h}$. The high light sensitivity of the camera of $0.05 \mathrm{~lx}$ enables the low-power excitation that limits the degradation after $4 \mathrm{~h}$ below $5 \%$ on average. Some of the polymers even showed enhanced emission by a few percent, presumably because of the removal of residues.

We performed fluorescence quenching experiments in dry air to study the detection reliability. The sensor array resides in air, followed by the consecutive delivery of target analytes at 1, 3, and $10 \mathrm{ppb}$. We further examined the sensor recovery by purging with dry air after the vapor exposure. Figure 4 depicts the observed QEs for each polymer per target class and sample set. Experimental details and sensor array recovery are given in the Supporting Information.

(a) P1

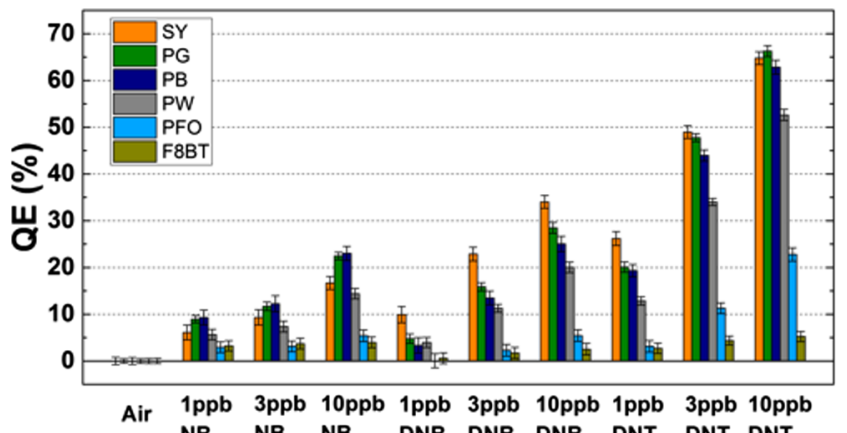

(b) P2

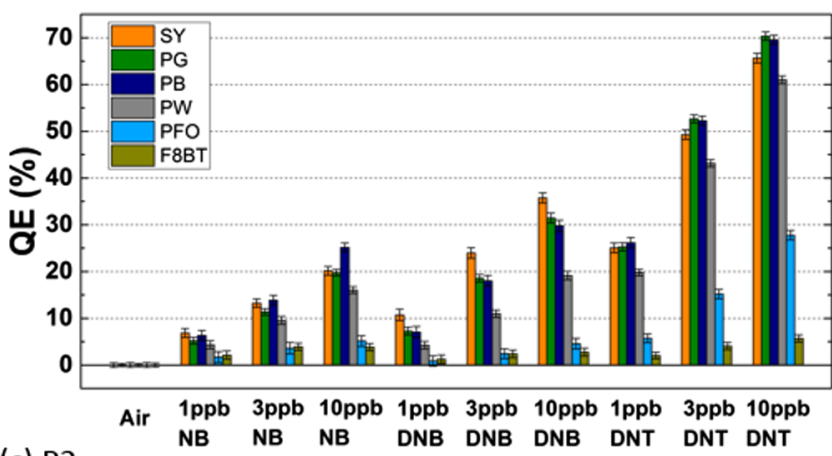

(c) P3

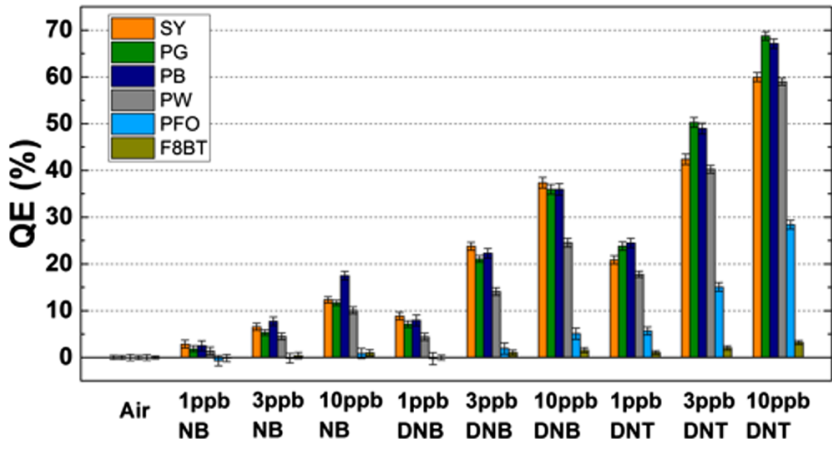

Figure 4. QE patterns across the three different sample sets: (a) P1, (b) P2, and (c) P3. The error bar definition and the polymer order correspond to that of Figure 3.

The QEs caused by DNT are up to $70 \%$ for $10 \mathrm{ppb}$, followed by up to almost $40 \%$ for $10 \mathrm{ppb} \mathrm{DNB}$ and up to $25 \%$ for 10 $\mathrm{ppb}$ NB. For each target class, there is a distinct response, that is, a fingerprint or a pattern. A minimum resolvable $\mathrm{QE}$ of $5 \%$ yields detection limits around $1 \mathrm{ppb}$ for DNT and DNB and around $3 \mathrm{ppb}$ for NB. Overall, the patterns appear similar to each other, and the sensor responses within each sample set are in good agreement as confirmed by the low QE standard deviations shown in Table 2. On average, P1 shows the largest standard deviations for the measured QEs, followed by P2 and $\mathrm{P} 3$, which is in line with the fluorescence appearance.

The application of a standardized LDA enables the discrimination of the target classes because of class separation. Figure 5 visualizes the transformed data, that is, the LDA training models, in two dimensions.

Here, LD1 and LD2 explain about 99\% of the data variability. Visual inspection of Figure 5 shows that the projections follow similar trajectories for the three sample sets. P2 and P3 are in 
Table 2. Average Standard Deviations (\%) of the QEs Across All Experiments

\begin{tabular}{ccccccc} 
& \multicolumn{7}{c}{ polymer sensor } \\
\cline { 2 - 7 } sample set & SY & PG & PB & PW & PFO & F8BT \\
P1 & 1.43 & 0.91 & 1.44 & 0.98 & 1.19 & 1.07 \\
P2 & 0.99 & 0.78 & 1.02 & 0.76 & 1.04 & 0.76 \\
P3 & 0.85 & 0.74 & 1.01 & 0.75 & 1.02 & 0.53 \\
\hline
\end{tabular}

(a) P1

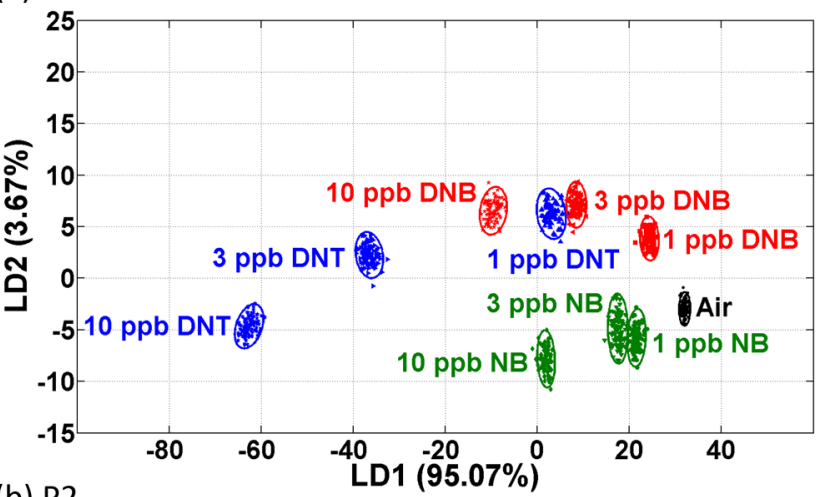

(b) P2

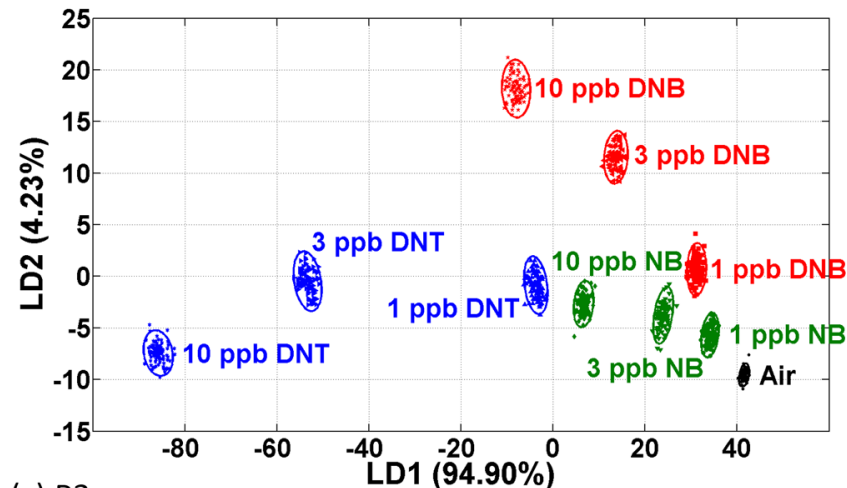

(c) P3

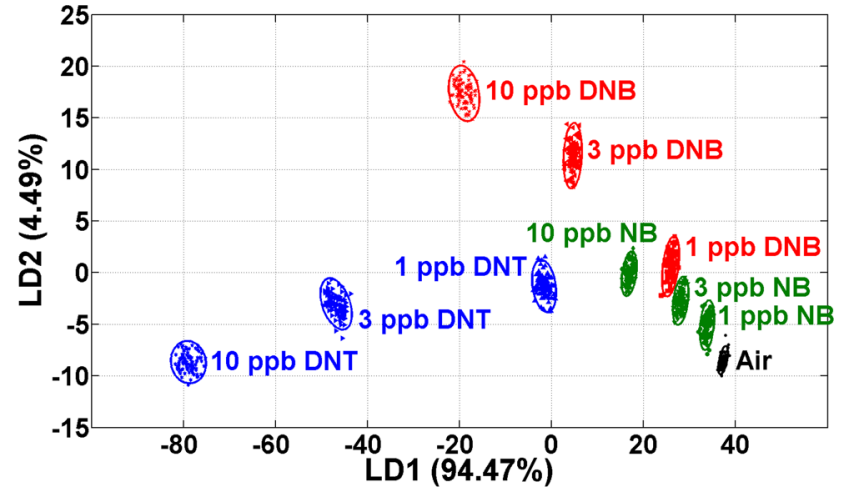

Figure 5. LDA training models in two dimensions for (a) P1, (b) P2, and (c) P3. The ellipses represent the 95\% confidence intervals for each target class. The explained variances are given in brackets.

good agreement with each other, whereas P1 seems to deviate slightly. This is particularly the case for NB and DNB, whereas DNT seems well-projected in P1. To study the reliability in terms of discriminatory reproducibility, the LDA training model of each set is used to classify the QE data from the other two sets, that is, the prediction of the target analyte and its concentration for each observation. Table 3 shows the accuracy rates for correct classifications without considering the confidence ellipses. This means, a classification will be
Table 3. Accuracy Rates (\%) for the Discriminatory Reproducibility

\begin{tabular}{clll} 
& \multicolumn{3}{c}{ test set } \\
\cline { 2 - 4 } training set & P1 & P2 & P3 \\
P1 & 100 & 81 & 45 \\
P2 & 81 & 100 & 84 \\
P3 & 61 & 96 & 100 \\
\hline
\end{tabular}

considered valid even if a projected observation with correctly classified class label is located outside a confidence ellipse.

As expected, the resubstitution accuracy is $100 \%$ for P1, P2, and P3 as the LDA training models show mostly nonoverlapping target separation in two dimensions already. The classification comparison of P2 versus P3 leads to excellent accuracy rates between 84 and 96\%, whereas P1 versus P2 shows high reproducibility of $81 \%$ but only low reproducibility between 45 and $61 \%$ for P1 versus P3. We attribute the poor reproducibility in the latter case to the significant differences in the fluorescence contributions from the outer ringlike structures. This is consistent with the visual differences in the LDA training models that are most pronounced between P1 and P3 in Figure 5a,c. A closer investigation of the specific confusion matrices, given in the Supporting Information, reveals more insights into these findings. The comparison of $\mathrm{P} 1$ versus $\mathrm{P} 3$ shows that there are no misclassifications for 10 and $3 \mathrm{ppb}$ DNT, which is in line with the visual inspection of the LDA training models. The prediction of some target classes for P1 versus P3, however, is of zero accuracy. The comparison of $\mathrm{P} 1$ versus $\mathrm{P} 2$ shows only few misclassifications, leading to the reported high accuracy rate of $81 \%$. The comparison of the highly reproducible sets P2 versus P3 with reported accuracy rates of $84-96 \%$ reveals that NB is the primary source of error in this case. This is in line with the small separation of the corresponding NB classes in the LDA training models. The prediction of DNT is of $100 \%$ accuracy, and the misclassifications for DNB are on a negligible scale such that P2 versus P3 shows excellent accuracy.

We attribute the excellent reproducibility of P1 versus P2 and P2 versus $\mathrm{P} 3$, corresponding to a twofold variation of the amount of the deposited material to the thickness independence in the center of the printed structures. This implies, however, that P1 versus P3 should be of similar reproducibility with accuracy rates between 81 and $96 \%$ because of the location of the ROIs that neglect the outer ringlike structures. The much poorer reproducibility of $\mathrm{P} 1$ versus $\mathrm{P} 3$ with accuracy rates of only $45-61 \%$, however, can be attributed to the fluorescence contributions from the outer ringlike structures that become much bigger for a fourfold variation of the amount of the deposited material. In reality, we would not expect such a strong variation in the fabrication process. Nevertheless, smaller ROIs in the centered plateau could be used to reduce the effect of contributions from the outer rings, but they would increase noise because of a lower number of pixels within the ROIs. A better solution would be the printing of larger sensor structures, for example, by the utilization of larger printer nozzles or by the utilization of a high-resolution camera. Overall, the accuracy rates remain remarkably high at $81-96 \%$ for a twofold variation of the amount of the deposited material, that is, P1 versus P2 and $\mathrm{P} 2$ versus $\mathrm{P} 3$.

Although we have demonstrated to discriminate trace nitroaromatic compounds using AJ printed polymeric sensor arrays and their reproducible and reliable fabrication, field 
applications require the sensor arrays to perform in complex matrices containing a series of potential interferents. Today, this aspect is another major challenge in the field of explosives detection and will be of future investigation.

\section{CONCLUSIONS}

We reported on the reliability in terms of discriminatory reproducibility of AJ printed fluorescence quenching sensor arrays by investigating the impact of variations in the amount of the deposited material. We observed high accuracy rates of more than $81 \%$ and up to $96 \%$ by doubling the amount of the deposited material. Thus, the results indicate a high reliability for the identification and quantification of explosive trace vapors close to the detection limits of a few ppb only. This demonstrates the promising potential of AJ printing for the fabrication of reliable fluorescence quenching sensor arrays. Moreover, the results underline the excellent capabilities of the LDA as a simple supervised statistical learning technique for the discrimination of explosive trace vapors.

\section{ASSOCIATED CONTENT}

\section{S Supporting Information}

The Supporting Information is available free of charge on the ACS Publications website at DOI: 10.1021/acsomega.7b01263.

Molecular structures of the target analytes, details on the measurement sequence and on the calculation of QEs, and the sensor array responses including recovery and the confusion matrices for the classification comparison of P1, P2, and P3 (PDF)

\section{AUTHOR INFORMATION}

\section{Corresponding Author}

*E-mail: nico.bolse@kit.edu (N.B.).

\section{ORCID $\odot$}

Nico Bolse: 0000-0002-7893-2743

Gerardo Hernandez-Sosa: 0000-0002-2871-6401

Uli Lemmer: 0000-0001-9892-329X

\section{Author Contributions}

All authors have contributed and have given approval to the final version of the manuscript.

Notes

The authors declare no competing financial interest.

\section{ACKNOWLEDGMENTS}

Financial support was gratefully received from the German Federal Ministry of Education and Research (BMBF) through grant FKZ 13N13691 and from the Karlsruhe School of Optics \& Photonics (KSOP).

\section{REFERENCES}

(1) Toal, S. J.; Trogler, W. C. Polymer Sensors for Nitroaromatic Explosives Detection. J. Mater. Chem. 2006, 16, 2871-2883.

(2) Rochat, S.; Swager, T. M. Conjugated Amplifying Polymers for Optical Sensing Applications. ACS Appl. Mater. Interfaces 2013, 5, 4488-4502.

(3) Thomas, S. W.; Joly, G. D.; Swager, T. M. Chemical Sensors Based on Amplifying Fluorescent Conjugated Polymers. Chem. Rev. 2007, 107, 1339-1386.

(4) Singh, S. Sensors-An Effective Approach for the Detection of Explosives. J. Hazard. Mater. 2007, 144, 15-28.

(5) Yinon, J. Field Detection and Monitoring of Explosives. Trends Anal. Chem. 2002, 21, 292-301.
(6) Meaney, M. S.; McGuffin, V. L. Luminescence-Based Methods for Sensing and Detection of Explosives. Anal. Bioanal. Chem. 2008, $391,2557-2576$

(7) Sun, X.; Wang, Y.; Lei, Y. Fluorescence Based Explosive Detection: from Mechanisms to Sensory Materials. Chem. Soc. Rev. 2015, 44, 8019-8061.

(8) Wang, Y.; La, A.; Ding, Y.; Liu, Y.; Lei, Y. Novel SignalAmplifying Fluorescent Nanofibers for Naked-Eye-Based Ultrasensitive Detection of Buried Explosives and Explosive Vapors. Adv. Funct. Mater. 2012, 22, 3547-3555.

(9) Räupke, A.; Palma-Cando, A.; Shkura, E.; Teckhausen, P.; Polywka, A.; Görrn, P.; Scherf, U.; Riedl, T. Highly Sensitive GasPhase Explosive Detection by Luminescent Microporous Polymer Networks. Sci. Rep. 2016, 6, 29118.

(10) Caron, T.; Guillemot, M.; Montméat, P.; Veignal, F.; Perraut, F.; Prené, P.; Serein-Spirau, F. Ultra Trace Detection of Explosives in Air: Development of a Portable Fluorescent Detector. Talanta 2010, 81, 543-548.

(11) Shanmugaraju, S.; Joshi, S. A.; Mukherjee, P. S. Fluorescence and Visual Sensing of Nitroaromatic Explosives Using Electron Rich Discrete Fluorophores. J. Mater. Chem. 2011, 21, 9130-9138.

(12) Meaney, M. S.; McGuffin, V. L. Investigation of Common Fluorophores for the Detection of Nitrated Explosives by Fluorescence Quenching. Anal. Chim. Acta 2008, 610, 57-67.

(13) Geng, Y.; Ali, M. A.; Clulow, A. J.; Fan, S.; Burn, P. L.; Gentle, I. R; Meredith, P.; Shaw, P. E. Unambiguous Detection of Nitrated Explosive Vapours by Fluorescence Quenching of Dendrimer Films. Nat. Commun. 2015, 6, 8240.

(14) Kose, M. E.; Harruff, B. A.; Lin, Y.; Veca, L. M.; Lu, F.; Sun, Y. P. Efficient Quenching of Photoluminescence from Functionalized Single-Walled Carbon Nanotubes by Nitroaromatic Molecules. J. Phys. Chem. B 2006, 110, 14032-14034.

(15) Hu, Z.; Deibert, B. J.; Li, J. Luminescent Metal-Organic Frameworks for Chemical Sensing and Explosive Detection. Chem. Soc. Rev. 2014, 43, 5815-5840.

(16) Germanenko, I. N.; Li, S.; El-Shall, M. S. Decay Dynamics and Quenching of Photoluminescence from Silicon Nanocrystals by Aromatic Nitro Compounds. J. Phys. Chem. B 2001, 105, 59-66.

(17) Nagarkar, S. S.; Joarder, B.; Chaudhari, A. K.; Mukherjee, S.; Ghosh, S. K. Highly Selective Detection of Nitro Explosives by a Luminescent Metal-Organic Framework. Angew. Chem., Int. Ed. 2013, 52, 2881-2885.

(18) Germain, M. E.; Knapp, M. J. Optical Explosives Detection: from Color Changes to Fluorescence Turn-On. Chem. Soc. Rev. 2009, $38,2543-2555$

(19) Yang, J.-S.; Swager, T. M. Porous Shape Persistent Fluorescent Polymer Films: An Approach to TNT Sensory Materials. J. Am. Chem. Soc. 1998, 120, 5321-5322.

(20) Johnston, J. M.; Williams, M.; Waggoner, L. P.; Edge, C. C.; Dugan, R. E.; Hallowell, S. F. Canine Detection Odor Signatures for Mine-Related Explosives. In Proceedings of the SPIE, Sept 4, 1998; Dubey, A. C., Harvey, J. F., Broach, J. T., Eds.; SPIE: Orlando, USA, 1998; Vol. 3392, p 490.

(21) Harper, R. J.; Almirall, J. R.; Furton, K. G. Identification of Dominant Odor Chemicals Emanating from Explosives for Use in Developing Optimal Training Aid Combinations and Mimics for Canine Detection. Talanta 2005, 67, 313-327.

(22) Furton, K. G.; Myers, L. J. The Scientific Foundation and Efficacy of the Use of Canines as Chemical Detectors for Explosives. Talanta 2001, 54, 487-500.

(23) Gazit, I.; Terkel, J. Explosives Detection by Sniffer Dogs Following Strenuous Physical Activity. Appl. Anim. Behav. Sci. 2003, 81, 149-161.

(24) Albert, K. J.; Walt, D. R. High-Speed Fluorescence Detection of Explosives-like Vapors. Anal. Chem. 2000, 72, 1947-1955.

(25) Woodka, M. D.; Schnee, V. P.; Polcha, M. P. Fluorescent Polymer Sensor Array for Detection and Discrimination of Explosives in Water. Anal. Chem. 2010, 82, 9917-9924. 
(26) Sohn, H.; Sailor, M. J.; Magde, D.; Trogler, W. C. Detection of Nitroaromatic Explosives Based on Photoluminescent Polymers Containing Metalloles. J. Am. Chem. Soc. 2003, 125, 3821-3830.

(27) Peveler, W. J.; Roldan, A.; Hollingsworth, N.; Porter, M. J.; Parkin, I. P. Multichannel Detection and Differentiation of Explosives with a Quantum Dot Array. ACS Nano 2016, 10, 1139-1146.

(28) Cho, J.; Anandakathir, R.; Kumar, A.; Kumar, J.; Kurup, P. U. Sensitive and Fast Recognition of Explosives Using Fluorescent Polymer Sensors and Pattern Recognition Analysis. Sens. Actuators, B 2011, 160, 1237-1243.

(29) Hughes, A. D.; Glenn, L. C.; Patrick, A. D.; Ellington, A.; Anslyn, E. V. A Pattern Recognition Based Fluorescence Quenching Assay for the Detection and Identification of Nitrated Explosive Analytes. Chem.-Eur. J. 2008, 14, 1822-1827.

(30) Diehl, K. L.; Anslyn, E. V. Array Sensing Using Optical Methods for Detection of Chemical and Biological Hazards. Chem. Soc. Rev. 2013, 42, 8596-8611.

(31) Askim, J. R.; Mahmoudi, M.; Suslick, K. S. Optical Sensor Arrays for Chemical Sensing: The Optoelectronic Nose. Chem. Soc. Rev. 2013, 42, 8649-8682.

(32) Ding, L.; Liu, Y.; Cao, Y.; Wang, L.; Xin, Y.; Fang, Y. A Single Fluorescent Self-Assembled Monolayer Film Sensor with Discriminatory Power. J. Mater. Chem. 2012, 22, 11574-11582.

(33) Miao, R.; Peng, J.; Fang, Y. Recent Advances in Fluorescent Film Sensing from the Perspective of both Molecular Design and Film Engineering. Mol. Syst. Des. Eng. 2016, 1, 242-257.

(34) Bolse, N.; Eckstein, R.; Schend, M.; Habermehl, A.; Eschenbaum, C.; Hernandez-Sosa, G.; Lemmer, U. A Digitally Printed Optoelectronic Nose for the selective trace Detection of Nitroaromatic Explosive Vapours Using Fluorescence Quenching. Flex. Print. Electron. 2017, 2, 024001.

(35) Bolse, N.; Eckstein, R.; Schend, M.; Habermehl, A.; HernandezSosa, G.; Eschenbaum, C.; Lemmer, U. Discrimination of Trace Nitroaromatics Using Linear Discriminant Analysis on Aerosol Jet Printed Fluorescent Sensor Arrays. In Proceedings of the SPIE, Apr 24, 2017; Baldini, F., Homola, J., Lieberman, R. A., Eds.; SPIE: Prague, Czech Republic, 2017; Vol. 10231, p 102310R. 